\title{
Processo Analítico Hierárquico (AHP) em ambiente SIG: temáticas e aplicações voltadas à tomada de decisão utilizando critérios espaciais
}

\author{
Analytical Hierarchical Process (AHP) in the GIS Environment: issues and \\ applications for decision making using spatial criteria
}

\section{Analyse Hiérarchique des Procédés (AHP) dans l'environnement SIG: thèmes et applications retour à la prise de décision en utilisant des critères spatiaux}

\author{
Proceso Analítico Jerárquico (AHP) en Ambiente SIG: temáticas y aplicaciones \\ volcadas a la toma de decisión utilizando criterios espaciales
}

\author{
Lianne Borja Pimenta ${ }^{1}$ \\ Norma Ely Santos Beltrão ${ }^{1}$ \\ Amanda Madalena da Silva Gemaque ${ }^{1}$ \\ Paulo Amador Tavares ${ }^{1}$ \\ Recebido em 23/02/2018; revisado e aprovado em 24/06/2018; aceito em 28/06/2018 \\ DOI: http://dx.doi.org/10.20435/inter.v20i2.1856
}

\begin{abstract}
Resumo: Trata-se de um levantamento bibliográfico sobre a aplicação do método AHP em combinação com Sistemas de Informações Geográficas (SIG). A revisão foi realizada com artigos publicados nos últimos 10 anos relacionados ao uso desse método, sob uma abordagem qualitativa. A partir da análise das 12 categorias científicas descritas neste artigo, foi possível apontar o método AHP usado com SIG como uma ferramenta eficaz para análise multicritério em diferentes cenários.
\end{abstract}

Palavras-chave: análise multicritério; desenvolvimento regional; política pública; Processo Analítico Hierárquico.

\begin{abstract}
This is a bibliographical survey about the application of the AHP method in combination with Geographic Information Systems (GIS). The review was carried out with articles published in the last 10 years related to the use of this method, under a qualitative approach. From the analysis of the 12 scientific categories described in this article, it was possible to point out the AHP method used with GIS as an effective tool for multicriteria analysis in different scenarios.
\end{abstract}

Keywords: multi-criteria analysis; regional development; public policy; Analytic Hierarchy Process.

Résumé: Ceci est une enquête bibliographique sur l'application de la méthode AHP en combinaison avec les SIG. La revue a été réalisée avec des articles publiés au cours des 10 dernières années liés à l'utilisation de cette méthode, sous une approche qualitative. À partir de l'analyse des 12 catégories décrites dans cet article, il a été possible de mettre en évidence la méthode AHP utilisée avec le SIG comme outil efficace pour l'analyse multicritère dans différents scénarios.

Mots-clé: analyse multicritere; developpement regional; politique publique; Analyse Hiérarchique des Procédés.

Resumen: Se trata de un levantamiento bibliográfico sobre la aplicación del método AHP en combinación con SIG. La revisión se realizó con artículos publicados en los últimos 10 años relacionados con el uso de este método, bajo un enfoque cualitativo. A partir del análisis de las 12 categorías científicas descritas en este artículo, fue posible apuntar el método AHP usado con SIG como una herramienta eficaz para análisis multicriterio en diferentes escenarios.

Palabras clave: análisis multicriterio; desarrollo regional; política pública; Proceso Analítico Jerárquico.

\footnotetext{
${ }^{1}$ Universidade do Estado do Pará (UEPA), Belém, Pará, Brasil.
} 


\section{INTRODUÇÃO}

A tomada de decisão considerando muitas variáveis é uma realidade em um contexto onde os problemas se tornam cada vez mais complexos, ao mesmo tempo que mais dados se tornam disponíveis. Problemas dessa natureza demandam, em geral, a avaliação de critérios representando atributos espaciais que possam assegurar uma decisão mais adequada à realidade local.

$\mathrm{Na}$ análise de problemas para a tomada de decisão, as informações obtidas através do levantamento de dados geram um elevado número de variáveis ou de atributos que, durante o processo de avaliação, necessitam de critérios aos quais são atribuídos pesos e valores. Assim, cria-se uma hierarquia em que, para facilitar a tomada de decisão, se prioriza os que detiverem maior peso e significância (FRANCO; HERNANDEZ; MORAES, 2013). Nesse processo, delimita-se o problema a ser solucionado e os atributos a serem considerados, para depois inserir quantificadores que permitem a estruturação das preferências dos tomadores de decisão (DROBNE; LISEC, 2009).

Assim, para dar suporte à tomada de decisão, com possibilidade de análise comparativa de fatores ou atributos, e de múltiplos cenários, têm surgido, a partir da década de 1960, os Métodos de Análise Multicritério (MAM), bastante utilizados em análises comparativas de projetos alternativos ou múltiplos objetivos (MEDEIROS, 2013).

Os MAM diferenciam-se entre si na especificação dos critérios que serão utilizados (escolha e níveis de preferência) e em como tais critérios e subcritérios serão categorizados ou hierarquizados, de maneira que, dependendo do objetivo, tem-se a possibilidade de um ou mais métodos para uso.

Dentre os métodos existentes, destacam-se o Preference Ranking Method for Enrichment Evaluation (PROMETHEE); Método Combinação Linear Ponderada (CLP) (Weighted Linear Combination-WLC); o Média Ponderada Ordenada (MPO); a Agregação pelo Operador (Ordered Weighted Averaging - OWA); o Elimination and Choice Translating Algorithm (ELECTRE), e o mais conhecido, o método Processo Analítico Hierárquico (Analytic Hierarchy Process - AHP) (FIGUEIRA; GRECO; EHRGOTT, 2005). A maioria desses métodos utiliza a modelagem matemática para harmonizar o processo de tomada de decisão, comparando alternativas ou cenários, de modo a apontar soluções mais adequadas aos interessados e/ou tomadores de decisão (SILVA; BELDERRAIN, 2005).

O objetivo deste artigo é apresentar um levantamento bibliográfico com abordagem exploratória sobre a aplicação do método AHP em combinação com o ambiente de Sistemas de Informações Geográficas (SIG). A partir da compreensão teórica e prática do método AHP, busca-se demonstrar os múltiplos usos encontrados na recente literatura científica e sua aplicabilidade na tomada de decisão de problemas envolvendo dados espaciais.

\section{ANÁLISE MULTICRITÉRIO COM FATORES ESPACIAIS}

Problemas ou planejamentos na área ambiental que envolvam análise espacial visando ao atendimento de um ou mais objetivos, geralmente utilizam um considerável agrupamento de variáveis múltiplas em ambiente SIG, caracterizando uma análise multicritérios (MALCZEWSKI, 2006).

A análise multicritérios com abordagem espacial têm sido comumente utilizada para planejamento do desenvolvimento, ocupação e uso da terra, no estudo e seleção de áreas aptas a 
diferentes usos (quais poderiam acarretar mais ou menos impactos perniciosos nos processos ambientais), em avaliações de impacto ambiental (previsões qualitativas desses processos), além da aptidão ou não de áreas para implementação de construções privadas e/ou públicas (COLLINS; STEINER; RUSHMAN, 2001).

A seleção de locais adequados para determinado fim é um problema clássico que tem utilizado os SIG como sua principal ferramenta, especialmente após a crescente oferta de softwares e linguagens de programação capazes de manusear as bases espaciais disponíveis nos mais diversos bancos de dados nacionais e internacionais. Para além dessa abordagem clássica de mapeamento SIG, tem-se a incorporação dos métodos MAM, sendo este último uma subdisciplina de pesquisa operacional que considera explicitamente múltiplos critérios em ambientes de tomada de decisão.

O MAM integrado ao SIG fornece procedimentos de solução mais adequados para esse problema, cuja seleção de locais seja baseada em critérios de decisão complexos e informações espaciais, uma vez que a seleção de locais adequados pode ser feita de forma mais abrangente com um conjunto de alternativas.

\section{O MÉTODO AHP COMO INSTRUMENTO DE TOMADA DE DECISÃO MULTICRITÉRIOS}

Entre os métodos de análise multicritérios mais difundidos e utilizados globalmente, o Processo Analítico Hierárquico ou em inglês Analytic Hierarchy Process (AHP) foi desenvolvido por Thomas L. Saaty na década de 1980, nos Estados Unidos. Trata-se de uma metodologia voltada para solução de problemas de escolha (opção), aplicada para diversas situações onde existam estruturas complexas. O método AHP analisa matematicamente comparações pareadas entre fatores em conjunto aos julgamentos e pesos de especialistas para avaliar critérios qualitativos ou intangíveis. Assim, são identificados fatores ou atributos que, quando selecionados, são organizados hierarquicamente descendentes de modo geral até o objetivo ou solução para o(os) problema(as) até o critério, subcritério e alternativas em diversos níveis (SAATY, 1990).

O método AHP tem sido utilizado em problemas espaciais de localização, na análise de adequabilidade de terras, através dos pesos que determinados critérios têm no alcance do objetivo da análise. Trata-se de um procedimento para os tomadores de decisão que aborda a aptidão de terras através da sistematização de critérios visando à seleção do local (CHANDIO et al., 2013). Nessa abordagem, os atributos são administrados e tratados em ambiente SIG, onde dados vetoriais ou matriciais convergem para a produção de um mapa (ou produto) final que irá evidenciar as potencialidades ou fragilidades para o embasamento da solução do problema (SAATY, 2005; 1990).

Para Saaty (1987), o método AHP é uma teoria geral de mensuração, usado para derivar escalas de comparações entre pares discretos e contínuos. Essas comparações podem ser tomadas a partir de medições reais ou de uma escala fundamental que reflete a força relativa das preferências e julgamentos. Inicialmente, decompõe-se hierarquicamente para então agregar as soluções de todos os subproblemas em uma conclusão. Esse processo facilita a tomada de decisões, organizando percepções, julgamentos e informações em uma estrutura que exibe as forças que influenciam uma decisão (SAATY, 1990). No caso simples e mais comum, as forças são organizadas a partir do mais geral e menos controlável para o mais específico e controlável, utilizando a capacidade humana inata de fazer julgamentos sobre pequenos problemas. 
O método AHP, conforme apresentado por Sousa et al. (2017), consiste em três operações principais, incluindo a construção de hierarquia, análise de prioridade e verificação de consistência, apesar de ser uma estrutura considerada normativa, ainda carrega um nível de subjetividade. Já Saaty (2008) argumenta que, mesmo a escala de preferência sendo considerada objetiva, o julgamento e as preferências por parte do decisor terão sempre um caráter subjetivo, já que estas possuem pesos proporcionais ao grau de importância dado a elas pelo tomador de decisão. Assim, os pesos serão atribuídos aos diferentes critérios e alternativas que caracterizam uma decisão, possibilitando escolher a melhor alternativa ao problema.

Para prosseguir com o processo de hierarquização proposto pelo método AHP, adaptou-se a escala absoluta de Saaty (1987), que contém a intensidade (de 1 a 9), definição e a justificativa de cada um dos itens, conforme apresentado no Quadro 1.

Quadro 1 - Escala Absoluta, Definição e Justificativa para o processo decisório com a AHP

\begin{tabular}{|c|c|c|}
\hline $\begin{array}{l}\text { Intensidade da } \\
\text { importância da } \\
\text { escala absoluta }\end{array}$ & Definição & Justificativa \\
\hline 1 & Igual importância & $\begin{array}{l}\text { As duas atividades contribuem equitativamente } \\
\text { para o objetivo }\end{array}$ \\
\hline 3 & $\begin{array}{l}\text { Importância moderada de um } \\
\text { sobre o outro fator }\end{array}$ & $\begin{array}{l}\text { Julgamento e experiência favorecendo } \\
\text { fortemente uma atividade sobre a outra }\end{array}$ \\
\hline 5 & Essencial ou forte importância & $\begin{array}{l}\text { Julgamento e experiência favorecendo } \\
\text { fortemente uma atividade sobre a outra }\end{array}$ \\
\hline 7 & Importância muito forte & $\begin{array}{l}\text { Uma atividade é fortemente favorecida e sua } \\
\text { dominância é demonstrada na prática }\end{array}$ \\
\hline 9 & Importância Extrema & $\begin{array}{c}\text { Evidência favorecendo uma atividade sobre a } \\
\text { outra é a mais expressiva possível na ordem de } \\
\text { afirmação }\end{array}$ \\
\hline $2,4,6,8$ & $\begin{array}{l}\text { Valores intermediários entre } \\
\text { os julgamentos adjacentes }\end{array}$ & Quando há necessidade de compromisso \\
\hline
\end{tabular}

Fonte: Adaptado de Saaty (1987).

Em muitos casos, a quantificação do julgamento do decisor entre dois critérios envolve dificuldades associadas com erros na mensuração dos atributos, imparcialidade nas avaliações e disponibilidade de informação, bem como imprecisões e ambiguidades inerentes ao procedimento da análise pareada (CHANG; CHANG; CHEN, 2009). Dessa forma, alguns estudos optam por utilizar a escala de relativa importância. A partir da escala absoluta, a qual atribui valores de 1 a 9, tem-se uma nova escala que determina a importância relativa de uma alternativa com a outra, facilitando o processo decisório. Inicialmente o avaliador deve julgar se o critério " $\mathrm{A}$ " é mais importante que o " $\mathrm{B}$ " (menos importante e mais importante) e, sendo este o caso, quanto " $A$ " é melhor que " $B$ ", onde se inserem os pesos utilizando-se a escala para quantificar essa percepção, como apresentado na Figura 1. 
Figura 1 - Escala relativa de Saaty utilizada para comparação pareada

$1 / 9$

Extremamente

$1 / 7$

$1 / 5$

Bastante Muito

\begin{abstract}
$1 / 3$
\end{abstract}
Pouco

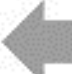

\section{Menos importante}

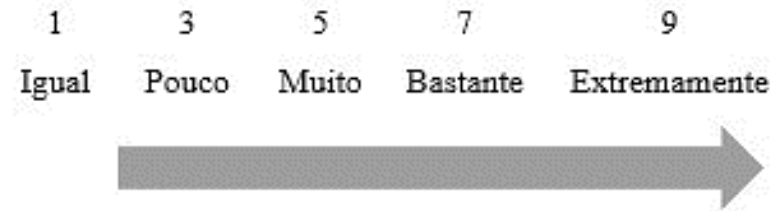

Mais importante

Fonte: Adaptado de Saaty (1987).

Pautando-se no modelo de Saaty (2008), a aplicação do método AHP é composta pelas seguintes atividades:

1. Definição dos critérios (mapas temáticos) que compõem a matriz de decisão hierárquica;

2. Construção do conjunto de matrizes de comparação pareada com os atributos selecionados na etapa anterior;

3. Atribuição de julgamentos/ pesos aos critérios previamente definidos, seja com base em dados previamente levantados (data-driven) ou por meio de painel de especialistas.

\section{METODOLOGIA DA PESQUISA}

O presente artigo apresenta uma revisão na literatura (bases de dados secundários) abrangendo artigos nacionais e internacionais relevantes publicados nos últimos 10 anos e que estivessem relacionados à aplicabilidade dos MAM's ao ambiente SIG - mais especificamente ao processo e metodologia pertinentes ao método AHP.

O levantamento bibliográfico enquanto metodologia de pesquisa foi utilizado nos estudos de Santos et al. (2017), Lima Júnior e Silva (2017) e Vieira et al. (2016), os quais se valeram da revisão integrativa da literatura e formulação de etapas para: decisão do tema utilizado; definição do problema ou questão norteadora; da seleção dos termos-chave a serem buscados nos artigos e periódicos e o tempo - período de tempo, que variou entre 4, 8 e 10 anos respectivamente. Artigos publicados anteriormente ao período escolhido não foram sumariamente excluídos por deterem conceitos-chave.

Palavras-chave buscadas compreenderam termos nos idiomas Português e Inglês, a fim de aumentar o raio e diversidade de artigos publicados, tais como: "AHP", "SIG", "Sensoriamento Remoto", "Análise espacial", "Analytic Hierarchy Process", "GIS", "Métodos de Decisão Multicritério", "Processo Analítico Hierárquico".

A pesquisa desenvolvida seguindo essas etapas garantiu a abordagem qualitativa deste trabalho - uma vez que permitiu uma análise sistêmica dos dados. Lara e Molina (2011) reforçam que a abordagem qualitativa não se embasa na rigidez de etapas e estrutura pertinentes à abordagem quantitativa e, também, que a revisão de literatura acerca do tema/problema seja aprofundada para permitir adequada discussão e desenvolvimento do estudo - não obstando o uso de dados quantitativos, que podem ser complementares à pesquisa. 


\section{RESULTADOS}

De acordo com a análise pretendida e do levantamento bibliográfico realizado, apresenta-se o Quadro 2 com objetivos, áreas e critérios de alguns trabalhos que utilizaram a AHP em ambiente SIG para solucionar seus respectivos problemas.

Quadro 2 - Categorias, autores, área de estudo e número de critérios

\begin{tabular}{|c|c|c|c|}
\hline Categorias & Autores & Área de Estudo & $\begin{array}{l}\text { Número de } \\
\text { critérios }\end{array}$ \\
\hline \multirow{2}{*}{$\begin{array}{l}\text { Planejamento } \\
\text { Urbano }\end{array}$} & $\begin{array}{l}\text { (1) Dai, Lee e Zhang } \\
\text { (2001) }\end{array}$ & $\begin{array}{l}\text { Área urbana do município de Lanzhou, } \\
\text { Noroeste da China. }\end{array}$ & 4 \\
\hline & (2) Lima et al. (2008) & $\begin{array}{l}\text { Área urbana do município de São Carlos, } \\
\text { São Paulo, Brasil. }\end{array}$ & 9 \\
\hline \multirow{2}{*}{$\begin{array}{l}\text { Deslizamento de } \\
\text { Terra }\end{array}$} & (3) Yalcin et al. (2011) & Município de Trabzon, nordeste da Turquia. & 7 \\
\hline & (4) Basu e Pal (2017) & Bacia hidrográfica de Gish, oeste da Índia. & 5 \\
\hline \multirow{2}{*}{ Turismo } & $\begin{array}{l}\text { (5) Bunruamkaew e } \\
\text { Murayama (2011) } \\
\end{array}$ & Província de Surat Thani, Tailândia. & 5 \\
\hline & $\begin{array}{l}\text { (6) Ghamgosar et al. } \\
\text { (2011) }\end{array}$ & $\begin{array}{l}\text { Área urbana do município de Langrood, } \\
\text { Irã. }\end{array}$ & 6 \\
\hline Irrigação & (7) Anane et al. (2012) & Região de Nabeul-Hammamet, Tunísia. & 5 \\
\hline $\begin{array}{c}\text { Serviços } \\
\text { Ecossistêmicos }\end{array}$ & $\begin{array}{l}\text { (8) Estoque e } \\
\text { Murayama (2012) } \\
\end{array}$ & $\begin{array}{l}\text { Área urbana do município de Baguio, } \\
\text { Filipinas. }\end{array}$ & 3 \\
\hline \multirow{2}{*}{$\begin{array}{l}\text { Agricultura/Uso da } \\
\text { terra }\end{array}$} & $\begin{array}{l}\text { (9) Akinci, Özalp e } \\
\text { Turgut (2013) }\end{array}$ & $\begin{array}{l}\text { Distrito de Yusufeli na cidade de Artvin, } \\
\text { Turquia. }\end{array}$ & 9 \\
\hline & $\begin{array}{l}\text { (10) Mishra, Deep e } \\
\text { Choudhary (2015) } \\
\end{array}$ & Município de Uttarakhand, Índia. & 6 \\
\hline $\begin{array}{c}\text { Desenvolvimento } \\
\text { Sustentável / } \\
\text { Encostas }\end{array}$ & $\begin{array}{l}\text { (11) Chandio et al. } \\
\text { (2014) }\end{array}$ & Município de George, Malásia. & 10 \\
\hline \multirow{2}{*}{$\begin{array}{c}\text { Construção de } \\
\text { estações de } \\
\text { combate a incêndio }\end{array}$} & (12) Wei et al. (2011) & Área urbana de Pequim, China. & 3 \\
\hline & $\begin{array}{l}\text { (13) Chaudhary et al. } \\
(2016)\end{array}$ & $\begin{array}{l}\text { Região metropolitana de Kathmandu, } \\
\text { Nepal. }\end{array}$ & 4 \\
\hline \multirow[b]{2}{*}{ Energia } & (14) Uyan (2013) & Região de Karapinar, Konya/Turquia. & 5 \\
\hline & $\begin{array}{l}\text { (15) Azevêdo, Candeias } \\
\text { e Tiba (2017) }\end{array}$ & Estado de Pernambuco. & 4 \\
\hline Risco de Inundação & (16) Roslee et al. (2017) & Área de Penampang, Sabah/Malásia. & 8 \\
\hline \multirow{3}{*}{ Recursos Hídricos } & $\begin{array}{l}\text { (17) Gdoura, Anane e } \\
\text { Jellali (2015) }\end{array}$ & $\begin{array}{l}\text { Área do aquífero raso de Nabeul- } \\
\text { Hammamet, Tunísia. }\end{array}$ & 6 \\
\hline & \begin{tabular}{|l}
$\begin{array}{l}\text { (18) Shekhar e Pandey } \\
\text { (2015) }\end{array}$ \\
\end{tabular} & $\begin{array}{l}\text { Distrito de Palamu, Estado de Jharkhand, } \\
\text { Índia. }\end{array}$ & 8 \\
\hline & (19) Pinto et al. (2017) & Bacia hidrográfica de Comoro, Timor Leste. & 8 \\
\hline Saúde & $\begin{array}{l}\text { (20) Camargo et al. } \\
\text { (2017) }\end{array}$ & Região Metropolitana de Campinas, Brasil. & 7 \\
\hline
\end{tabular}

Fonte: Autores (2018). 
Os critérios utilizados nos estudos apresentados demonstram o caráter espacial da seleção deles pelos autores, visto que a maioria dos critérios se alinham com dados de distribuição espacial e de localização. O quadro 3 apresenta a diversidade de critérios utilizados na literatura consultada, especificando os resultados da presente revisão.

Quadro 3- Autores e critérios encontrados na revisão

\begin{tabular}{|c|c|}
\hline Autor & Critérios Utilizados \\
\hline $\begin{array}{l}\text { Dai, Lee e Zhang } \\
\text { (2001) }\end{array}$ & opografia; Condições do Solo; Lençóis Freáticos; Risco Geológico. \\
\hline Lima et al. (2008) & $\begin{array}{l}\text { Volume de Tráfego e Classificação Funcional; Tipo de Rota; Capacidade } \\
\text { Funcional; Capacidade Estrutural; Idade; Localização; Questões de Segurança; } \\
\text { Questões Ambientais; Fatores Econômicos. }\end{array}$ \\
\hline Yalcin et al. (2011) & $\begin{array}{l}\text { Geologia; Declividade; Aspecto; Elevação; Cobertura da Terra; Proximidade ao } \\
\text { Sistema ou Rede de Drenagem e Distância de Estradas. }\end{array}$ \\
\hline Basu e Pal (2017) & $\begin{array}{l}\text { Fatores Desencadeantes; Fatores Litológicos; Fatores de Superfície; Fatores } \\
\text { Antropogênicos; Fatores Protetivos. }\end{array}$ \\
\hline $\begin{array}{l}\text { Bunruam } \\
\text { Murayam }\end{array}$ & $\begin{array}{l}\text { Paisagem; Vida Selvagem; Topografia; Acessibilidade; Características da } \\
\text { Comunidade. }\end{array}$ \\
\hline $\begin{array}{l}\text { Ghamgosar et al. } \\
(2011)\end{array}$ & Aspecto; Solo; Rocha; Uso do Solo. \\
\hline Anane et a & $\begin{array}{l}\text { Classes de Capacidade de Uso da Terra; Conflitos de Recursos; Econômico; } \\
\text { Ambiental. }\end{array}$ \\
\hline $\begin{array}{r}\text { Estoque e } \\
\qquad(20\end{array}$ & nto Populac \\
\hline $\begin{array}{l}\text { Akinci e Özalp e } \\
\text { Turgut (2013) }\end{array}$ & $\begin{array}{l}\text { Classes de Solo; Classes de Capacidade de Uso da Terra; Sub-classes de } \\
\text { Capacidade de Uso da Terra; Profundidade do Solo; Erosão; Outras Propriedades } \\
\text { do Solo; Declividade; Aspectos (Irradiação); Elevação. }\end{array}$ \\
\hline $\begin{array}{r}\text { Mishra, } \\
\text { Choudha }\end{array}$ & renagem; Rodovias; Solo; Geologia; Uso e Cobertura da Terra; Declividade. \\
\hline Chandio et al. (2014) & $\begin{array}{l}\text { Rodovia Primária, Rodovia Secundária, Elevação, Declividade, Aspecto, Terras } \\
\text { Agrícolas, Área Florestal, Residência Existente, Terras Alagadas, Águas superficiais }\end{array}$ \\
\hline Wei et al. (2011) & $\begin{array}{l}\text { Densidade Populacional; Perdas após Incêndios Prediais; Distância da Estação } \\
\text { de Combate a Incêndio mais Próxima. }\end{array}$ \\
\hline $\begin{array}{l}\text { Chaudhary et al. } \\
\text { (2016) }\end{array}$ & $\begin{array}{l}\text { Distâncias das Rodovias; Cobertura da Terra; Distância dos Rios; Densidade } \\
\text { Populacional. }\end{array}$ \\
\hline Uyan & $\begin{array}{l}\text { Distância de Áreas Residenciais; Uso da Terra; Distância de Rodovias; Declividade; } \\
\text { Distância de Linhas de Transmissão. }\end{array}$ \\
\hline $\begin{array}{l}\text { Azevêdo, Candeias e } \\
\text { Tiba (2017) }\end{array}$ & limático; Topografia; Ambiental; Localidade. \\
\hline Roslee (2017) & $\begin{array}{l}\text { Precipitação; Gradiente de Declividade; Elevação; Densidade de Drenagem; Uso } \\
\text { da Terra; Texturas do Solo; Curvas de Declividade; Acúmulo de Fluxo }\end{array}$ \\
\hline $\begin{array}{l}\text { Gdoura, Anane e } \\
\text { Jellali (2015) }\end{array}$ & $\begin{array}{l}\text { Textura do Solo; Geologia; Elevação; Uso da Terra; Distância de Áreas Urbanas; } \\
\text { Qualidade da Água Subterrânea. }\end{array}$ \\
\hline $\begin{array}{l}\text { Shekhar e Pandey } \\
\text { (2015) }\end{array}$ & $\begin{array}{l}\text { Geomorfologia; Litologia; Solo; Declividade; Densidade Linear; Densidade de } \\
\text { Drenagem; Pluviosidade; Espessura da Zona de Resistência. }\end{array}$ \\
\hline Pinto et al. (2017) & $\begin{array}{l}\text { Densidade Linear; Uso da Terra; Declividade; Topografia; Solo; Litologia; } \\
\text { Densidade de Drenagem; Precipitação. }\end{array}$ \\
\hline $\begin{array}{l}\text { Camargo et al. } \\
(2017)\end{array}$ & $\begin{array}{l}\text { Iluminação Pública; Pavimentação; Calçamento; Arborização; Esgoto a Céu } \\
\text { Aberto; Lixo Acumulados; Rede Geral Distribuição de Água. }\end{array}$ \\
\hline
\end{tabular}

Fonte: Autores (2018). 


\subsection{Planejamento urbano, infraestrutura e energia}

No trabalho desenvolvido por Lima et al. (2008), foi delineado um estudo que utilizou um método de análise multicritério em ambiente SIG, para auxiliar no desenvolvimento de um modelo de gestão para ações prioritárias de conservação de vias/pavimentos, na área urbana do município de São Carlos, São Paulo, Brasil. Para a aplicação da AHP, foi necessário selecionar critérios relevantes no processo de análise e decisão. Os resultados em forma de índices de ações prioritárias enfatizaram que as áreas contidas nas faixas de "0.4-0.6" e "0.6-0.8" foram as que obtiveram maior peso, logo maior necessidade de reparos.

O planejamento para a construção de novas estações de combate a incêndios na área que compreende os anéis rodoviários, mais especificamente o terceiro, na área urbana de Pequim, China foi objeto do estudo de Wei et al. (2011). Com o intuito de reduzir a incerteza na identificação de áreas mais favoráveis ou adequadas, os autores utilizaram o método AHP juntamente ao ambiente SIG, selecionando três critérios. Na produção do mapa final houve a ocorrência de áreas que obtiveram, no mapa e na legenda, a coloração "Vermelha", "Verde" e "Azul" como as regiões de importância progressiva, respectivamente.

Chaudhary et al. (2016) também desenvolveram um estudo para identificação de potencial e favorabilidade para construção de estações de combate a incêndios na região metropolitana de Katmandu, no Nepal. Com a seleção de quatro critérios trabalhados em ambiente SIG junto com o método AHP, foi possível produzir um mapa final que apontou que aproximadamente 13,46\% da área total da cidade encontrava-se classificada como "Altamente favorável" para a construção.

Outra aplicabilidade encontrada para associação do método AHP e ambiente SIG se refere à identificação de áreas com potencial para a instalação de usinas de energia solar para o Estado de Pernambuco, desenvolvido por Azevêdo et al. (2017). Alguns dos subcritérios encontrados na matriz de comparação eram similares aos critérios utilizados em outros trabalhos, são eles: "Declividade, Uso do solo, Distância de corpos hídricos e Áreas urbanas". Áreas adequadas ou com favorabilidade à instalação foram reclassificadas em "Alta" e "baixa", com faixas de transição nas cores, tanto no mapa como na legenda.

\subsection{Análise de riscos: deslizamento de terra e Inundações}

Yalcin et al. (2011) buscaram mapear em seu estudo as áreas suscetíveis ao deslizamento de terra no município de Trabzon, nordeste da Turquia, visando à redução de uma das maiores causas de morte do país. Para tal, foi utilizada a AHP em conjunto ao ambiente SIG, através da seleção de sete critérios ou parâmetros, que, colocados na matriz de comparação, foram hierarquizados. Com isso, delimitou-se subcritérios e obtiveram-se os pesos para que se viabilizasse, com os dados espaciais, a produção de um mapa apontando a propensão ou não de determinadas áreas para a ocorrência de deslizamentos. A reclassificação no mapa final foi de "Muito baixa, Baixa, Moderada, Alta e Muito alta suscetibilidade".

Já o estudo desenvolvido por Roslee et al. (2017) tencionava avaliar o nível e potencial de Inundação na área de Penampang, Sabah, na Malásia. Para isso, utilizaram o método AHP (e suas respectivas etapas) aliado ao ambiente SIG, para apontar quais áreas seriam mais propensas à inundação e quais não o seriam, a fim de orientar o planejamento urbano e mitigar os efeitos da inundação na área de estudo. Assim, foram selecionados oito critérios considerados importantes e influentes no processo. A reclassificação dos mapas ficou em "Alta" e "Baixa", com faixas de transição observadas no mapa e nas legendas. 


\subsection{Agricultura e uso sustentável da terra}

O trabalho desenvolvido por Mishra et al. (2015) no município de Uttarakhand, na Índia, objetivava identificar o potencial para desenvolvimento de agricultura orgânica visando à melhoria econômica da área, utilizando o método multicritério AHP em conjunto ao ambiente SIG. Os dados espaciais de seis critérios foram trabalhados e padronizados no software ArcGIS - dando origem a um mapa final com as reclassificações "Muito mais favorável, Mais, Menos e Não-favorável" ao desenvolvimento de agricultura orgânica.

Outra aplicação refere-se à combinação de uso do método AHP ao ambiente SIG, desenvolvido no estudo de Chandio et al. (2014), cuja abordagem tencionava apontar o potencial de favorabilidade para o uso sustentável das áreas de encosta devido à escassez de terras planas na Ilha de Penang, município de George, Malásia. Notas dadas pelos especialistas e a matriz de comparação foram trabalhados no software Expert Choice (EC), além da verificação do índice de consistência (que faz parte da análise de consistência do julgamento/atribuição das notas). Nos mapas de cada um de seus critérios, a reclassificação ficou em "Altamente, Moderado, Pouco ou Inadequado" para o potencial das áreas.

\subsection{Recursos hídricos}

Pinto et al. (2017) integraram ao desenvolvimento do seu estudo o método AHP, o ambiente SIG e dados de sensoriamento remoto para a delimitação de zonas e seus potenciais para exploração de águas subterrâneas de modo sustentável na Bacia Hidrográfica de Comoro, em Timor Leste. Utilizando oito critérios, produziu-se o mapa final, gerado no ArcGIS 10.1, com a reclassificação definida em "Muito pobre, Pobre, Moderado, Alto e Muito alto".

Outro estudo, desenvolvido no distrito de Palamu, no Estado de Jharkhand, na Índia, também buscou a delineação de áreas para exploração de águas subterrâneas. Shekhar e Pandey (2015) utilizaram a mesma associação AHP, SIG e Sensoriamento Remoto, para produzir um mapa final reclassificando o potencial para "Excelente, Muito bom, Bom, Moderado, Fraco e Muito fraco". De acordo os autores, aproximadamente $88 \%$ do território estudado não têm potencial favorável à exploração.

\subsection{Saúde e turismo}

No trabalho de Buruamkaew e Murayama (2011), a utilização do método multicritério AHP aliado ao ambiente SIG, objetivou a identificação da favorabilidade para a priorização de áreas para o ecoturismo na província de Surat Thani, na Tailândia. Os pesos foram calculados no Microsoft Excel, e mapas no formato raster foram produzidos no software ArcGIS 9.3, os quais foram manejados para a produção dos mapas de favorabilidade. No mapa final, a identificação das áreas com potencial para ecoturismo na província apresentava a seguinte classificação "Alta, Moderada, Levemente ou Não adequado".

Outro estudo valeu-se de objetivo similar, e visou indicar o potencial de áreas para o desenvolvimento do ecoturismo na área do distrito de Langrood, Irã, aplicando-se o método AHP em consonância ao ambiente SIG (GHAMGOSAR et al., 2011). Nesse trabalho, foram utilizados seis critérios considerados importantes para a análise.

A combinação do método AHP em ambiente SIG também tem sua aplicabilidade no auxílio do processo de gestão da saúde pública, como demonstra um estudo realizado na Região 
Metropolitana de Campinas, São Paulo, Brasil, desenvolvido por Camargo et al. (2017). Neste, o objetivo era identificar a potencialidade de áreas que integravam a Região Metropolitana de Campinas suscetíveis à contaminação por doenças em decorrência da ausência de saneamento básico. Para a produção de um mapa final que evidenciasse tal possibilidade, foram utilizados sete critérios com as faixas de reclassificação definidas em "Muito baixa, Baixa, Média e Alta" para a favorabilidade de acordo com os setores zoneados na análise.

O levantamento bibliográfico apresentado no Quadro 2 evidenciou a versatilidade e a viabilidade de aplicação do método AHP em diversas temáticas que requerem uso de dados espaciais. Trata-se, portanto, de um método que, ao produzir um mapa final com suas reclassificações e potenciais, é capaz de reduzir as incertezas de processos decisórios complexos e que envolvem diversos fatores (socioambientais, econômicos e políticos) - complexidade essa, próxima dos muitos desafios relacionados ao planejamento ambiental e de uso da terra no contexto brasileiro.

\section{CONCLUSÃO}

Dentro do amplo espectro de métodos de análise multicritérios existentes, é possível apontar o caráter prático do método AHP, além de sua viabilidade e versatilidade na redução de incertezas. O processo de análise hierárquica de múltiplos critérios para tomada de decisão possui elementos capazes de avaliar as potencialidades ou restrições inerentes ao processo decisório, influenciando positivamente no planejamento. Com isso, dispõe-se de um importante subsídio à formulação de políticas públicas mais específicas e possivelmente mais eficazes, voltadas ao desenvolvimento socioambiental e econômico.

A partir dos trabalhos selecionados através de levantamento bibliográfico, foi possível identificar 12 categorias científicas com aplicação distintas, com a utilização de 122 critérios. Todos os trabalhos utilizaram o método AHP em consonância ao ambiente SIG, e apresentaram estudos realizados em diferentes países.

Apesar de certa subjetividade presente em parte do processo de análise hierárquica AHP (seleção, preferência dos critérios e julgamento pelos especialistas), tal processo tem sido bastante disseminado academicamente e publicado em revistas com fator de impacto importante (no indexador Scopus).

Assim, no contexto do ambiente SIG, o uso do método AHP para identificar áreas com riscos, potencial ou favorabilidade, poderia contribuir na melhoria da gestão dos recursos ambientais. Ademais, configura-se como uma ferramenta capaz de auxiliar na formulação de políticas públicas; em ações de combate ao desmatamento; na proteção de unidades de conservação; no planejamento urbano; ações de segurança pública; saúde, entre outros - promovendo um desenvolvimento mais equilibrado.

Como recomendação final, e no âmbito de aplicação para políticas públicas e promoção de um desenvolvimento regional mais sustentável, sugere-se o emprego do método AHP com dados espaciais em ambiente SIG, na análise e avaliação das condições de favorabilidade para expansão da fronteira agrícola no estado do Pará. Trata-se de um desafio para o planejamento regional, tendo em vista a complexidade e extensão de área a ser analisada, o que requer a avaliação de muitos fatores e critérios, muitos dos quais apresentados em bases espaciais que necessitam ser processadas em ambiente SIG. Essa aplicação faz parte do mesmo projeto de pesquisa que originou este artigo e será apresentado posteriormente. 


\section{REFERÊNCIAS}

AKINCI, H.; ÖZALP, A. Y.; TURGUT, B. Agricultural land use suitability analysis using GIS and AHP technique. Computers and Electronics in Agriculture, v. 97, p. 71-82, set. 2013. Disponível em: https://www. sciencedirect.com/science/article/pii/S0168169913001567

ANANE, M.; BOUZIRI, L.; LIMAM, A.; JELLALI, S. Ranking suitable sites for irrigation with reclaimed water in the Nabeul-Hammamet region (Tunisia) using GIS and AHP-multicriteria decision analysis. Resources, Conservation and Recycling, v. 65, p. 36-46, ago. 2012. Disponível em: https://www.sciencedirect.com/ science/article/pii/S0921344912000730

AZEVÊDO, V. W. B.; CANDEIAS, A. L. B.; TIBA, C. Location study of solar thermal power plant in the state of Pernambuco using geoprocessing technologies and multiple-criteria analysis. Journal Energies, v. 10, n. 7, 2017. Disponível em: https://www.mdpi.com/1996-1073/10/7/1042

BASU, T.; PAL, S. Exploring landslide susceptible zones by analytic hierarchy process (AHP) for the Gish River Basin, West Bengal, India. Spatial Information Research. v. 25, n. 5, p. 665-75, out. 2017.

BUNRUAMKAEW, K.; MURAYAMA, Y. Site Suitability Evaluation For Ecotourism Using GIS \& AHP: a case study of Surat Thani Province, Thailand. Procedia Social and Behavioral Sciences, v. 21, p. 269-78, 2011. Disponível em: https://www.sciencedirect.com/science/article/pii/S1877042811013474

CAMARGO, D. M.; SOUZA, N. M.; PACHECO, T. C. K. F.; ALCÂNTARA, G. C.; DOTA, E. M. Modelagem geoespacial para identificação de áreas vulneráveis ao contágio por doenças relacionadas a falta de saneamento: o caso da Região Metropolitana de Campinas. Revista Brasileira de Cartografia, v. 69, n. 3, p. 561-73, mar. 2017. Disponível em: http://www.seer.ufu.br/index.php/revistabrasileiracartografia/ article/view/44349/23431

CHANDIO, I. A.; MATORI, A. N. B.; WANYUSOF, K. B.; TALPUR, M. A. H. T.; BALOGUN, A.-L.; LAWAL, D. U. GISbased analytic hierarchy process as a multicriteria decision analysis instrument: a review. Arabian Journal of Geosciences, v. 6, n. 8, p. 3059-66, ago. 2013. Disponível em: https://link.springer.com/article/10.1007/ s12517-012-0568-8

CHANDIO, I. A.; MATORIB, A. N.; YUSOFB, K.; TALPUR, M. A. H.; AMINU, M. GIS-basedland suitability analysis of sustainable hillside development. Procedia Engineering, v. 77, p. 87-94, 2014. Disponível em: /pii/ https://www.sciencedirect.com/science/article/pii/S1877705814009862

CHANG, N.; CHANG, Y.; CHEN, H. Fair fund distribution for a municipal incinerator using GIS-based fuzzy analytic hierarchy process. Journal of Environmental Management, v. 90, n. 1, p. 441-54, 2009. Disponível em: https://www.sciencedirect.com/science/article/pii/S0301479707004082

CHAUDHARY, P.; CHHETRI, S. K.; JOSHI, K. M.; SHRESTHA, B. M.; KAYASTHA, P. Application of an Analytic Hierarchy Process (AHP) in the GIS interface for suitable fire site selection: a case study from Kathmandu Metropolitan City, Nepal. Socio-Economic Planning Sciences, v. 53, p. 60-71, mar. 2016. Disponível em: https://www.sciencedirect.com/science/article/pii/S0038012115000452

COLLINS, M. G.; STEINER, F. R.; RUSHMAN, M. J. Land-use suitability analysis in the United States: historical development and promising technological achievements. Environmental Management, v. 28, n. 5, p. 611-21, 2001

DAI, F. C.; LEE, C. F.; ZHANG, X. H. GIS-based geo-environmental evaluation for urban land-use planning: a case study. Engineering Ggeology, v. 61, n. 4, p. 257-71, set. 2001. Disponível em: https://www. sciencedirect.com/science/article/pii/S001379520100028X 
DROBNE, S.; LISEC, A. Multi-attribute decision analysis in gis: weighted linear combination and ordered weighted averaging. Informatica, v. 33, n. 4, p. 459-74, jan. 2009.

ESTOQUE, R. C.; MURAYAMA, Y. Examining the potential impact of land use/cover changes on the ecosystem services of Baguio city, the Philippines: a scenario-based analysis. Applied Geography, v. 35, n. 1-2, p. 31626, nov. 2012. Disponível em: https://www.sciencedirect.com/science/article/pii/S0143622812000872

FIGUEIRA, J.; GRECO, S.; EHRGOTT, M. Multiple criteria decision analysis: state of art - surveys. International series in operations research \& management science. New York: Springer International Editions - Verlag, 2005.

FRANCO, R. A. M.; HERNANDEZ, F. B. T.; MORAES, J. F. L. O uso da análise multicritério para a definição de áreas prioritárias a restauração de Área de Preservação Permanente (APP), no noroeste paulista. In: SIMPÓSIO BRASILEIRO DE SENSORIAMENTO REMOTO - SBSR, 16., 2013, Foz do Iguaçu-PR. Anais [...]. Foz do Iguaçu, PR: INPE, 2013.

GDOURA, K.; ANANE, M.; JELLALI, S. Geospatial and AHP-multicriteria analyses to locate and rank suitable sites for groundwater recharge with reclaimed water. Resources, Conservation and Recycling, v. 104, parte A, p. 19-30, nov. 2015. Disponível em: https://www.sciencedirect.com/science/article/pii/ S0921344915300811

GHAMGOSAR, M.; HAGHYGHY, M.; MEHRDOUST, F.; ARSHAD, N. Multicriteria decision making based on Analytical Hierarchy Process (AHP) in GIS for tourism. Middle-East Journal of Scientific Research, v. 10, n. 4, p. 501-7, 2011. Disponível em: https://pdfs.semanticscholar.org/0b4a/486f62e0b56c2138f9fcf5ae4c 647ad1bb11.pdf

LARA, A. M. B.; MOLINA, A. A. Pesquisa Qualitativa: apontamentos, conceitos e tipologias. In: TOLEDO, C. A. A.; GONZAGA, M. T. C. (Org.). Metodologia e técnicas de pesquisa nas áreas de ciências humanas. Maringá, PR: EdUem, 2011. v. 01, p. 121-72.

LIMA JÚNIOR, P.; SILVA, S. G. Educação em ciência e democracia: uma revisão de literatura em periódicos internacionais. In: CONGRESO INTERNACIONAL SOBRE INVESTIGACIÓN EN DIDÁCTICA DE LAS CIENCIAS, 10., 5-8 set. 2017, Sevilla, Espanha. Anais [...]. Sevilla, Espanha, 2017. Disponível em: https://ddd.uab. cat/pub/edlc/edlc_a2017nEXTRA/16_-_Educacao_em_Ciencia_e_Democracia.pdf

LIMA, J. P.; FERNANDES JÚNIOR, J. L.; RAMOS, R. A. R.; LIMA, R. S. Processo hierárquico analítico na gestão da conservação de vias urbanas pavimentadas. In: ENCONTRO NACIONAL DE ENGENHARIA DE PRODUÇÃO- A integração de cadeias produtivas com a abordagem da manufatura sustentável, 28., 2008, Rio de Janeiro. Anais [...]. Rio de Janeiro, 2008.

MALCZEWSKI, J. GIS-based multicriteria decision analysis: a survey of the literature. International Journal of Geographical Information Science, v. 20, n. 7, p. 703-26, 2006.

MEDEIROS, A. Análise multicritério em SIG - Parte 1. 2013. Disponível em: http://www.andersonmedeiros. com/analise-multicriterio-em-sig-1/. Acesso em: 15 maio 2017.

MISHRA, A. K.; DEEP, S.; CHOUDHARY, A. Identification of suitable sites for organic farming using AHP \& GIS. The Egyptian Journal of Remote Sensing and Space Sciences, v. 18, n. 2, p. 181-93, dez. 2015. Disponível em: https://www.sciencedirect.com/science/article/pii/S1110982315000289

PINTO, D.; SHRESTHA, S.; BABEL, M. S.; NINSAWAT, S. Delineation of groundwater potential zones in the Comoro watershed, Timor Leste using GIS, remote sensing and analytic hierarchy process (AHP) technique. Applied Water Science, v. 7, n. 1, p. 503-19, mar. 2017. Disponível em: https://link.springer. com/article/10.1007/s13201-015-0270-6 
ROSLEE, R.; TONGKUL, F.; SIMON, N.; NORHISHAM, M. N. Flood Potential Analysis (FPAn) using Geo-Spatial Data in Penampang area, Sabah. Malaysian Journal Geosciences (MJG), v. 1, n. 1, p. 1-6, 2017.

SAATY, T. L. Decision making with the analytic hierarchy process. International Journal of Services Sciences, v. 1, n. 1, p. 83-98, jan. 2008.

SAATY, T. L. The analytic hierarchy and analytic network processes for the measurement of intangible criteria and for decision-making. In: FIGUEIRA, J.; GRECO, S.; EHRGOTT, M. (Ed.). Multiple criteria decision analysis: state of the art-surveys. Boston/Dordrecht/London: Kluwer Academic Publishers, 2005. Cap. 9.

SAATY, T. L. How to make a decision: the analytic hierarchy process. European Journal of Operational Research, v. 48, n. 1, p. 9-26, 1990. Disponível em: https://www.sciencedirect.com/science/article/ pii/0377221790900571

SAATY, R. W. The analytic hierarchy process - what it is and how it is used. Mathematical Modelling, v. 9, n. 3-5, p. 161-76, 1987. Disponível em: https://www.sciencedirect.com/science/article/pii/0270025587904738

SANTOS, L. E. S.; SANTOS, J. S.; RIBEIRO, A. C. O.; NUNES, I. T. O.; BARROS, F. D. Cuidados de enfermagem voltados a pacientes com Acidente Vascular Encefálico: uma Revisão Integrativa de Literatura. Congresso Internacional de Enfermagem, v. 1, n. 1, p. 1-3, 2017. Disponível em: https://eventos.set.edu.br/index. $\mathrm{php/cie/article/view/6111.}$

SHEKHAR, S.; PANDEY, A. C. Delineation of groundwater potential zone in hard rock terrain of India using remote sensing, geographical information system (GIS) and analytic hierarchy process (AHP) techniques. Journal Geocarto International, v. 30, n. 4, p. 402-21, 2015.

SILVA, R. M.; BELDERRAIN, M. C. N. Considerações sobre métodos de decisão multicritério. In: ENCONTRO DE INICIAÇÃO CIENTíFICA E PÓS-GRADUAÇÃO DO ITA, 11., 2005. Anais [...]. São José dos Campos: XI Encita, 2005. p. 1-7. Disponível em: http://www.bibl.ita.br/xiencita/Artigos/Mec03.pdf 2005.

SOUSA, J. V.; JERÔNIMO, T. B.; MELO, F. J. C.; AQUINO, J. T. Uso do AHP para identificação de perdas da qualidade em empresas de manufatura: um estudo de caso. Exacta, São Paulo, v. 15, n. 1, p. 89-100, 2017. Disponível em: http://periodicos.uninove.br/index.php?journal=exacta\&page=article\&op=view\& path\%5B\%5D=6691

UYAN, M. GIS-based solar farms site selection using Analytic Hierarchy process (AHP) in Karapinarregion, Konya/ Turkey. Renewable and Sustainable Energy Reviews, v. 28, p. 11-7, dez. 2013. Disponível em: https://www. sciencedirect.com/science/article/pii/S1364032113004875

VIEIRA, N. R. S.; DANTAS, R. A. N.; DANTAS, D. V.; SANTOS, J. J. S.; VASCONCELOS, E. F. L.; CARVALHO, I. C. T. Caracterização da produção científica sobre intoxicações exógenas: revisão integrativa da literatura. Revista Saúde, Guarulhos, SP, v. 10, n.1-2, p. 47-60, 2016. Disponível em: http://revistas.ung.br/index. php/saude/article/view/2152

WEI, L.; LI, H.; LIU, Q.; CHEN, J.; CUI, Y. Study and implementation of fire sites planning based on GIS and AHP. Procedia Engineering, v. 11, p. 486-95, 2011.

YALCIN, A.; REIS, S.; AYDINOGLU, A. C.; YOMRALIOGLU, T. A GIS-based comparative study of frequency ratio, analytical hierarchy process, bivariate statistics and logistics regression methods for landslide susceptibility mapping in Trabzon, NE Turkey. Catena, v. 85, n. 3, p. 274-87, jun. 2011. Disponível em: https://www. sciencedirect.com/science/article/pii/S0341816211000233 


\section{Sobre autores:}

Lianne Borja Pimenta: Mestranda em Ciências Ambientais pela Universidade do Estado do Pará (UEPA). E-mail: lianneborja@yahoo.com.br, Orcid: http://orcid.org/0000-0003-3961-0183

Norma Ely Santos Beltrão: Doutora em Economia Agrícola pela Justus-Liebig-Universität Giesse. Professora adjunta na Universidade do Estado do Pará (UEPA).E-mail: normaelybeltrao@gmail.com, Orcid: http://orcid.org/0000-0003-1991-2977

Amanda Madalena da Silva Gemaque: Doutoranda em Ciências Ambientais pela Universidade Federal do Pará (UEPA). E-mail: amanda.gemaque@yahoo.com.br, Orcid: http://orcid.org/0000-0002-3809-7931

Paulo Amador Tavares: Mestrando em Ciências Ambientais pela Universidade do Estado do Pará (UEPA). E-mail: atavares.paulo@gmail.com, Orcid: http://orcid.org/0000-0003-2617-1548 